\title{
First-in-man feasibility study of a novel ingestible magnetically inflated balloon capsule for treatment of obesity
}

\section{다(1) $(9)$}

\author{
Authors \\ Institutions \\ 1 Department of General Surgery, National University \\ Hospital, Singapore \\ 2 School of Mechanical \& Aerospace Engineering, \\ Nanyang Technological University, Singapore \\ 3 Department of Medicine, National University of \\ Singapore
}

Hung Leng Kaan¹, Phuoc Thien Phan², Anthony Meng Huat Tiong², Muneaki Miyasaka², Soo Jay Phee², Khek Yu Ho ${ }^{3}$

submitted 19.6.2019

accepted after revision 30.12.2019



Corresponding author

Professor Khek Yu Ho, Department of Medicine, Yong Loo Lin School of Medicine, National University of Singapore, NUHS Tower Block, 1E Kent Ridge Road, Level 11, Singapore
119228, Singapore

Fax: +65-6779 4112

mdchoky@nus.edu.sg

\section{ABSTRACT}

Intragastric balloons (IGBs) are an established treatment option for obesity. Major barriers to dissemination of IGBS include lack of long-term efficacy outcomes, safety concerns, cost, and tolerability. We developed a novel ingestible magnetically inflated balloon capsule (IMI-BC) in hopes of overcoming these challenges. The IMI-BC is significantly cheaper than IGBs currently available on the market. We performed proof-of-concept animal studies and a first-inhuman feasibility study to demonstrate the feasibility of inflating the IMI-BC using an external magnet. Further studies are currently being conducted to evaluate the safety, tolerability, and long-term efficacy of the IMI-BC. When fully developed, we anticipate that this device will benefit obese patients.

\section{Introduction and background}

Incidence of obesity is increasing rapidly worldwide. Multiple studies have shown that it is a major risk factor for cardiovascular morbidity and mortality [1]. Despite the success of bariatric surgery, obese patients are at increased risk of anesthetic adverse events (AEs) as preoperative comorbidities are relatively common among this group of patients [2]. Because of this, a minimally-invasive alternative, the intragastric balloon (IGB), has gained popularity.

Recent studies have demonstrated the safety and efficacy of IGBs [3]. However, most IGBs require liquid to be introduced into the balloon via a catheter, hence the need for catheter and/or endoscopic assistance for insertion and/or removal of the balloon. We believe that an ideal IGB should be inflated and deflated without the need for endoscopic or catheter assistance. We have developed an IMI-BC that can be inflated via external magnetic control without onboard electronics. We performed successful proof-of-concept studies on the IMI-BC using a virtual stomach EMS Trainer and fresh porcine stomachs [4].
The aims of the current study were twofold: (1) to demonstrate efficacy of the original wireless mechatronic capsule IGB prototype in inducing weight loss in a surviving animal; and (2) to examine the feasibility of inflating the balloon of the latest ingestible microcapsule IGB prototype using an external magnet in an animal and a human volunteer.

\section{Methods}

\section{Devices}

We initially designed a wireless mechatronic capsule ( $\varnothing 19 \times$ $50 \mathrm{~mm}$ ) that consisted of an enclosure and a balloon that stored acid and base respectively (U.S. Patent 9375554, 2016) [5]. It also contained on-board electronics including battery and communication unit ( $\mathbf{F i g} \mathbf{1}$ ). When the electronics received command wirelessly, the linear actuator pushed the acid into the balloon to react with the base, thus inflating the balloon. The wireless mechatronic balloon capsule is able to inflate to a volume of $500 \mathrm{~mL}$. 


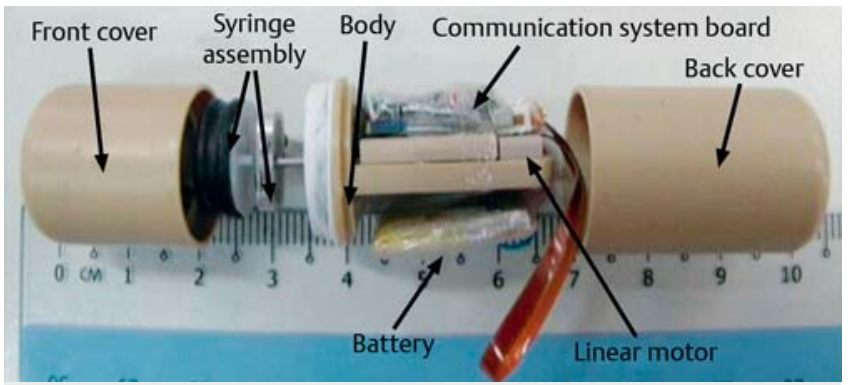

Fig. 1 Configuration of the wireless capsule.

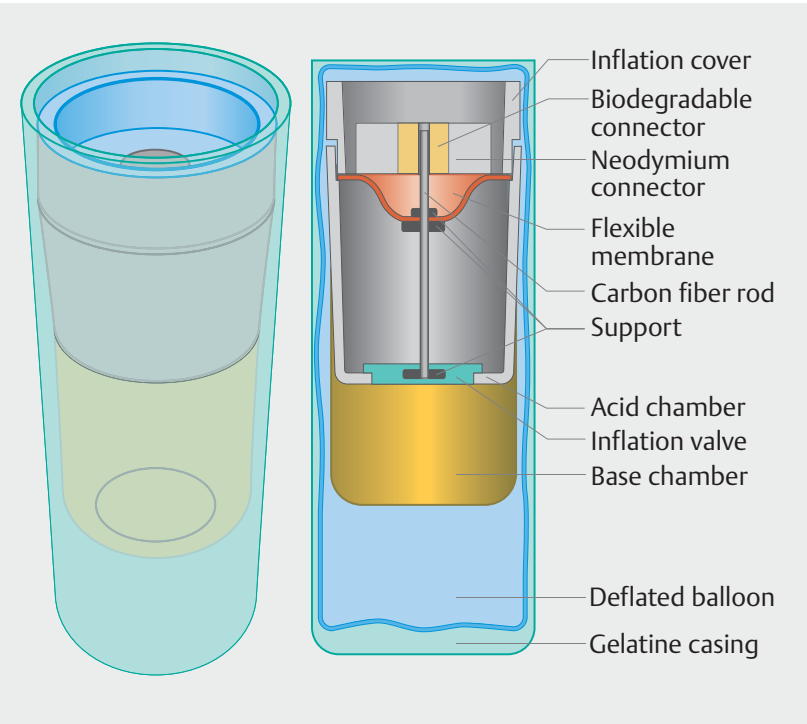

Fig. 2 Configuration of IMI-BC.

The latest prototype has no onboard electronics ( $\triangleright$ Fig. 2). The device is housed inside a gelatin casing with a diameter and length of $13 \mathrm{~mm}$ and $35 \mathrm{~mm}$, respectively ( $>$ Table 1 ). Magnetic sensors are used to confirm that the device has reached the stomach for safe inflation. Upon triggering the inflation actuator, citric acid and potassium bicarbonate in separate chambers interact and release carbon dioxide immediately. The balIoon of the IMI-BC is able to inflate to a volume of $120 \mathrm{~mL}$ within 6 minutes.

\section{Survival animal trial: mechatronic wireless capsule IGB}

This animal survival study was designed to evaluate weight loss after placement of the mechatronic wireless capsule IGB. It involved the use of six live adult female pigs weighing 35 to $40 \mathrm{~kg}$ each. Given the limited funding and time available, we administered the wireless capsule IGB to only one of the pigs which weighed $37.8 \mathrm{~kg}$. The other five pigs served as controls.

\section{Procedure}

Under general anaesthesia, the wireless capsule IGB was delivered into the stomach of one of the pigs endoscopically. A radiofrequency signal was sent from an external unit to activate a linear actuator onboard the capsule, releasing the acetic acid to interact with the sodium bicarbonate, hence generating carbon dioxide to inflate the balloon. The wireless balloon capsule was inflated to a volume of $500 \mathrm{~mL}$. The inflated balloon was left in the pig's stomach for a week and the pig was fed the same feed as five other control pigs. The treated pig was observed for a week before being weighed again and then it was euthanized. Necropsy was performed to check for treatmentrelated $\mathrm{AEs}$.

\section{Outcome measures}

The main outcome measure was change in weight following administration of the wireless capsule IGB. We measured the weights of the treated and control pigs prior to the start of the trial, and again at 1 week post-procedure to determine if any weight loss had occurred. The secondary outcome measure was AEs related to the procedure.

\section{Non-survival animal trial: magnetically actuated capsule IGB (IMI-BC)}

This was a preclinical non-survival animal trial to demonstrate the feasibility of inflating two IMI-BCs in one pig using an externally-controlled magnet.

\section{Procedure}

One adult male pig weighing $31.9 \mathrm{~kg}$ was used for the study. The animal was fed a normal diet until 3 days prior to the procedure and subsequently fasted for 12 hours. The animal was euthanatized prior to the experiment to save cost of the general

- Table 1 IMI-BC characteristics.

\begin{tabular}{|l|l|}
\hline Characteristic & Potential benefits \\
\hline Compact microscapsule $(13 \mathrm{~mm} \times 35 \mathrm{~mm})$ & $\begin{array}{l}\text { Likely easily ingestible and may not require insertion via endoscopy. } \\
\text { Multiple capsules can be ingested gradually over several days to achieve the desired space occupying } \\
\text { effect while avoiding the side effect of nausea and vomiting due to sudden inflation }\end{array}$ \\
\hline Inflation mechanism and base chamber & $\begin{array}{l}\text { Valve is easily opened using a hand-held external magnet, allowing for acid-base interaction to release } \\
\text { carbon dioxide for inflation of the balloon }\end{array}$ \\
\hline Gelatin casing & Expanding balloon easily breaks the gelatin casing \\
\hline Balloon made of biodegradable materials & $\begin{array}{l}\text { Upon degradation of the balloon, it will deflate automatically and hence allow self-excretion of IMI-BC } \\
\text { without the need for endoscopy for retrieval }\end{array}$ \\
\hline
\end{tabular}


anesthesia. The IMI-BC was delivered endoscopically and activated via an external handheld magnet. The procedure was repeated with the delivery and inflation of the second IMI-BC. We dissected the stomach to visualize the two inflated intragastric balloons.

\section{Outcome measures}

The main outcome measures were: (1) time required for each of the balloons to start inflating upon activation by the external hand-held magnet; (2) time required for the balloons to be fully inflated; (3) volumes of inflation; and (4) presence or absence of leak of the balloons.

\section{First-in-human feasibility study: magnetically actuated IGB (IMI-BC)}

We then conducted a first-in-man feasibility study to evaluate the magnetically-controlled balloon inflation mechanism of the ingestible weight loss capsule in one human subject.

\section{Eligibility criteria and recruitment of subject}

One human volunteer was recruited for the study. Inclusion criteria included being a healthy subject aged between 21 to 50 years old with no gastrointestinal symptoms or previous gastrointestinal surgery. Exclusion criteria included being a pregnant female; having anatomical, congenital or functional abnormalities of the upper gastrointestinal tract; having bleeding diathesis; and having previous implants or bioenteric devices.

\section{Procedure}

The subject, a female aged 41 years, was fasted 6 hours prior to the procedure and subsequently sedated. The IMI-BC was delivered endoscopically. A magnetic sensor was used to confirm its location prior to activation via an external handheld magnet. Once the balloon had been fully inflated, the subject was observed for a further one minute to identify any immediate adverse events. Subsequently, the balloon was punctured with an injection needle and retrieved via a snare.

\section{Outcome measures}

The main outcome measures were: (1) time required for the balloon to start inflating upon activation by the external handheld magnet; and (2) time required for the balloon to be fully inflated.

\section{Results}

\section{Survival animal trial: mechatronic wireless capsule IGB}

After 1 week, the pig with the wireless capsule in its stomach lost $1.5 \mathrm{~kg}$, from $37.8 \mathrm{~kg}$ before to $36.3 \mathrm{~kg}$ after placement of the wireless capsule. The five control pigs gained a range of 3 to $5 \mathrm{~kg}$ over a period of 2 weeks. There was no sign of distress nor complication in any of the pigs during the 1-week observation period. After euthanasia, the balloon was noted to remain inflated with the volume of $110 \mathrm{~mL}$ and the capsule was intact.


- Fig. 3 a Dimensions of IMI-B. b Insertion of IMI-BC and inflation of the balloon using an externally controlled magnet. c Inflated intragastric balloon seen via endoscopy within a live euthanized pig's stomach.

\section{Non-survival animal trial: magnetically actuated capsule IGB (IMI-BC)}

For the non-survival study, the first IMI-BC was successfully delivered. It inflated within 15 seconds after activation by the hand-held magnet, and was fully inflated within 6 minutes. ( Fig.3). The second IMI-BC was also successfully deployed and activated to inflate within 15 seconds. However, it became deflated before it could achieve full inflation. At the end of the animal trial, we retrieved the two IMI-BCs by cutting into the pig's stomach. There was no leak in the first balloon. Its volume of inflation was $120 \mathrm{~mL}$. In the second balloon, suboptimal sealing was noted, thus explaining the failure to achieve full inflation of the balloon.

\section{First-in-human feasibility study: magnetically actuated IGB (IMI-BC)}

The IMI-BC was successfully deployed endoscopically into the subject's stomach (\$Fig.4). The magnetic sensors were able to confirm the device's location. There was successful inflation of the microcapsule's balloon within 1 minute to a full inflation volume of approximately $120 \mathrm{~mL}$ within 3 minutes ( $\mathbf{F i g . 5}$ ). For the next 1 minute of observation, the balloon remained fully inflated. The patient's vital signs were recorded to be normal throughout the procedure. 


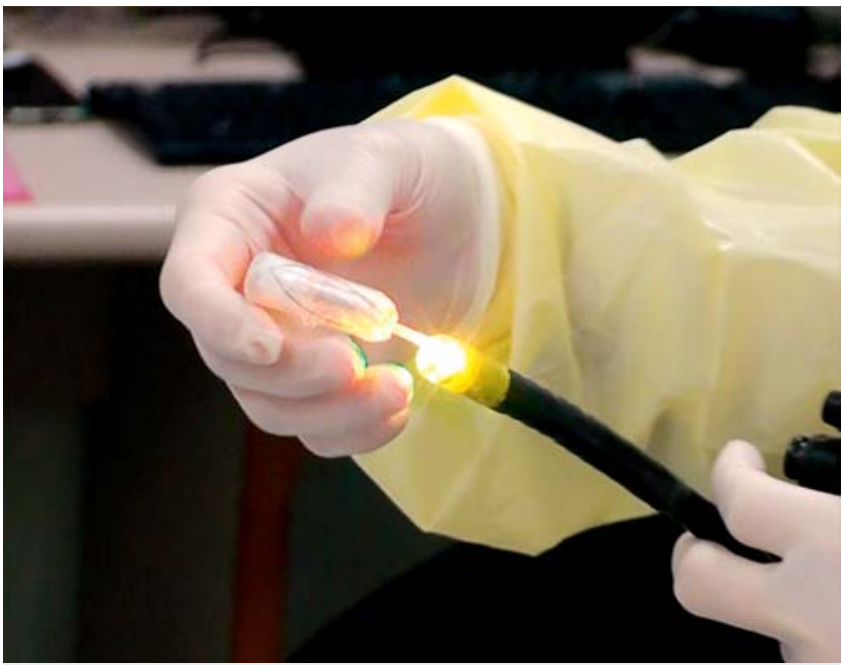

Fig. 4 Introduction of IMI-BC via a gastroscope.

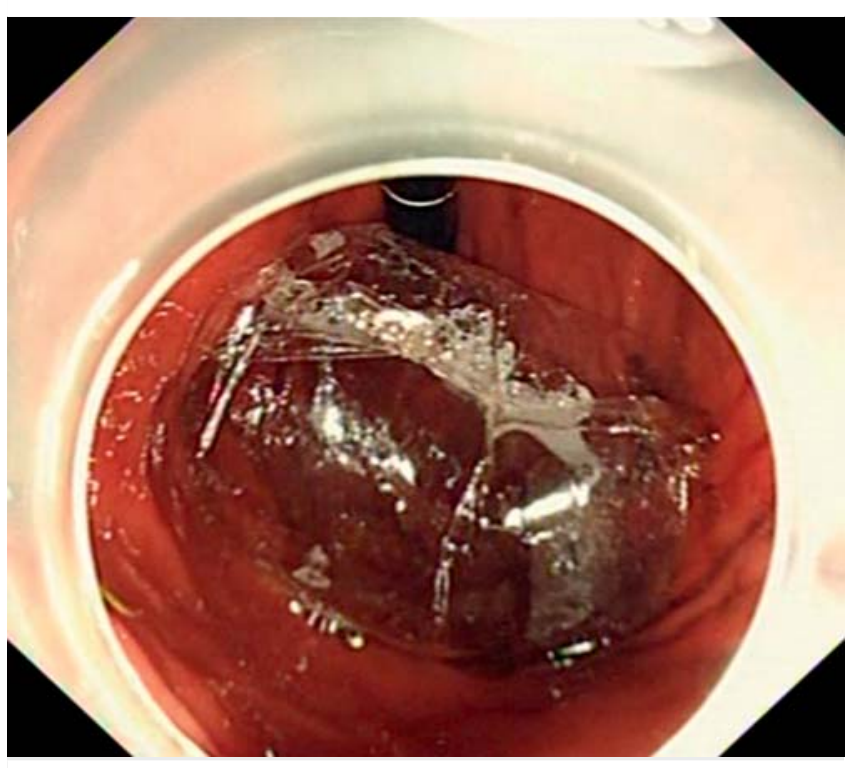

- Fig.5 Inflated intragastric balloon seen via endoscopy within a human stomach.

\section{Discussion}

For an IGB to be widely used in the clinical setting, it should have efficacy in inducing weight loss, safety in administration, and ease of use. While current IGBs have proven themselves to be effective and safe in weight management, most require use of a catheter to inflate the balloon with liquid and endoscopy to insert and/or retrieve it. In doing do, the procedures need sedation or anesthesia, thereby increasing the overall cost of therapy. The IMI-BC that we have developed is able to overcome these usability issues.

The IMI-BC only requires a simple external hand-held magnet to activate its inflation valve within the chamber, initiating a chemical reaction to release the carbon dioxide, which inflates the balloon. This removes the need for a catheter and injection of saline or water to inflate the balloon. For safety pur- poses, an external magnetic sensor is used to confirm that the IMI-BC is within stomach prior to inflation. Absence of onboard electronics is attractive in terms of cost-effectiveness. These advantages potentially allow the IMI-BC to be easily administered by doctors in the hospital setting, and even within the outpatient primary care setting.

There are certain limitations in our trials, which we plan to address in subsequent studies. First, given the limited funds and time available, we started off by performing small-scale animal and human studies. In our subsequent studies, we plan to perform larger-scale animal and human trials to allow us to draw appreciable conclusions regarding the efficacy and safety of our device.

In our survival animal trial, the pig with the wireless capsule in the stomach lost a significant amount of weight. With the larger-scale animal trials, we aim to evaluate mean weight loss and demonstrate sustained weight loss post-procedure. Second, as the aims of the studies above were mainly to demonstrate feasibility, we will investigate in future trials the optimal volume and number of capsules required, and duration of their inflation to achieve sustained weight loss. Third, we aim to further investigate the safety of our device, ensuring that the balloon can be deflated safely and excreted through the gastrointestinal tract without causing small bowel obstruction. We aim to construct the balloon using a biodegradable material, allowing carbon dioxide to be self-excreted, hence aiding the balloon in deflation. We also aim to enhance our location sensor to tracking the capsule's migration along the gastrointestinal system. Our future human trials will then aim to confirm that the device can be swallowed without endoscopic assistance for insertion, externally activated to inflate in the stomach, left inflated in the stomach for a few days, naturally deflated, and excreted through the gastrointestinal system.

\section{Competing interests}

The authors declare that they have no conflict of interest.

\section{References}

[1] Whitlock G, Lewington S, Sherliker P et al. Body-mass index and cause-specific mortality in 900000 adults: collaborative analyses of 57 prospective studies. Lancet 2009; 373: 1083-1096

[2] Genco A, Lorenzo M, Baglio G et al. Does the intragastric balloon have a predictive role in subsequent LAP-BAND ${ }^{\circledR}$ surgery? Italian multicenter study results at 5-year follow-up Surg Obes Relat Dis 2014; 10 : 474-478

[3] Ponce J, Quebbemann BB, Patterson EJ. Prospective, randomized, multicenter study evaluating study and efficacy of intragastric dualballoon in obesity. Surg Obes Relat Dis 2013; 9: 290-295

[4] Do TN, Seah TET, Ho KY et al. Development and testing of a magnetically actuated capsule endoscopy for obesity treatment. PLoS ONE 2016; 11: e0148035

[5] Phee SJ, Wong KJ, Tan SL et al. Balloon inflating device and a method for inflating a balloon. U.S. Patent 9375554. 28.06.2016 